\title{
Preparation of Novel Lignophenol-based Polymers using Cellulose Fibers as Vanishing Reaction-Supports
}

\author{
Mitsuru Aoyagi, Satoko Yonekura, Chika Miyasaka and Masamitsu Funaoka \\ Graduate School of Bioresources, Mie University, Japan Science and Technology Agency (JST)-Solution Oriented Research for \\ Science and Technology (SORST), 1577 Kurimamachiya-Cho, Tsu, 514-8507, Japan \\ Fax: +81-59-231-9591, e-mail: aoyagi@bio.mie-u.ac.jp
}

\begin{abstract}
Two syntheses of novel lignophenol (LP) derivatives via hydroxymethlylated (HM) lignophenol ( $p$-cresol type) under conditions of the phase-separation system were carried out. First, brush-type derivatives were carried out using HM-Western Hemlock (Tsuga Heterophilla)-lignophenol ( $p$-cresol type, HMLC1) as benzyl compounds. Resulting derivatives were obtained with high yields. Due to high reactivity and high affinity between $p$-cresol and HMCL1, there are no influences with or without reacting supports. Resulting LC2, LC3 and LC4 showed almost same characteristic properties as LP estimated by ${ }^{1} \mathrm{H}-\mathrm{NMR}$, TMA, GPC. Secondly, bulky alkyl phenols were also tried to apply for second grafting phenols to HM-Hinoki cypress (Chamaecyparis obtusa)-lignophenol ( $p$-cresol type, HM-HCLC1). Without vanishing reaction-supports (pulp, freeness100), all derivatives were aggregated. On the other hand, using reaction-supports, 4-sec-buthylphenol (4SBP), 4-(1,1,3,3-tetramethyl)buthyl phenol (4TMBP) and 4-pentylphenol(4PP) generated derivatives with $47.6 \%, 25.4 \%$ and $59.4 \%$ yields, respectively. A part of derivatives were dissolved into purification solvents such as EtOEt. Resulting derivatives were estimated by GPC, TMA and ${ }^{1} \mathrm{H}-\mathrm{NMR}$.

Key words: lignin, lignophenol, benzyl structure, cellulose, reaction support
\end{abstract}

\section{INTRODUCTION}

Recently serious environmental problems such as global warming concerning exhaustion of $\mathrm{CO}_{2}$ have been paid a lot of attentions. Moreover apprehension for exhaustion of petroleum has also been attracted. To overcome a part of these problems, utilization of biomass has been tried for substitution of petroleum as both fuel and materials because of its sustainability. Especially lignocellulosics such as wood, glasses were regarded as one of best substitutions. However it has been almost impossible to be utilized whole lignocellulosics after separation of contents due to complicated and enforced inter-penetrating complexes (IPN structures) of both lignin and carbohydrates[1]. Therefore in an aspect of material utilization, only carbohydrates have been utilized as pulps, which amounts are corresponded to only $50 \%$ of materials, in industry. As chemical reactivity of lignin was exhausted through the separation process under high temperature and pressure, lignin has been utilized as only fuel.

In order to utilize whole lignocellulosics, new reaction system, the phase-separation system, with both separations and structural conversions which consist of heterogeneous surface reactions has been developed [2-4]. Through the surface reactions between phenol/ concentrated acids, both carbohydtares and lignin are separated and converted into aqueous carbohydrates and

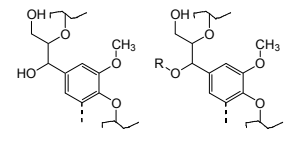

Softwood Native Lignins (Benzyl / C $\alpha$-O-ether type linkages)

Fig.1 Initial reactions with $p$-cresol of benzyl and $\mathrm{C} \alpha$ - $O$-ether type structures in native lignin under acidic conditions through the phase -separation system. lignin-based polymers, lignophenols (LPs), quantitatively under $1 \mathrm{~atm}$ and room temperature.

Through the reaction, at first step, 3-dimensional linkages of native lignin were quickly released on both benzyl aryl ether structures or benzyl units which included about 5-10\%[5] in softwood under acidic conditions[6-7]. However, main structures, $\mathrm{C} \beta-O-4$ arylether linkages were not cleft. On the cleavages benzyl aryl ethers, rich phenols around native lignin selectively attacked to $\mathrm{C} \alpha$ carbocations. The resulting lignin-based polymers have 1,1-bis(aryl)propane-2-O-arylether-3-ol type structures with rich $\mathrm{C} \beta-O-4$ linkages about $80 \%[4,8]$.

On the other hand, benzyl structures are good reaction sites for nucleophillic attacks due to stable benzyl cations because of high reactivity especially under both acidic and heated conditions. For example, benzyl alcohol easily reacted to aliphatic-OH under only $\mathrm{pH}=6$ buffer at $60{ }^{\circ} \mathrm{C}$ with self-condensations [9-11].

In fact, catechol was easily grafted to hydroxymethylated Hinoki cypress-lignophenol ( $p$-cresol type, HCLC-HM) through the phase-separation system $\left(72 \% \mathrm{H}_{2} \mathrm{SO}_{4}\right)$ with almost $100 \%$ of yields without condensations, although catechol without a reaction-support was easily dissolved and self-condensed under strong acid condition [12]. In general, lignin-based materials are likely to be aggregated and then self-condensed by itself. Therefore, to prevent from aggregating, cellulose reaction supports were used as vanishing reaction-supports through the phase-separation system. These reaction-supports acted to keep heterogeneous reaction surfaces with degrading by hydrolysis gradually [12].

Therefore using benzyl structures of HM structures, 


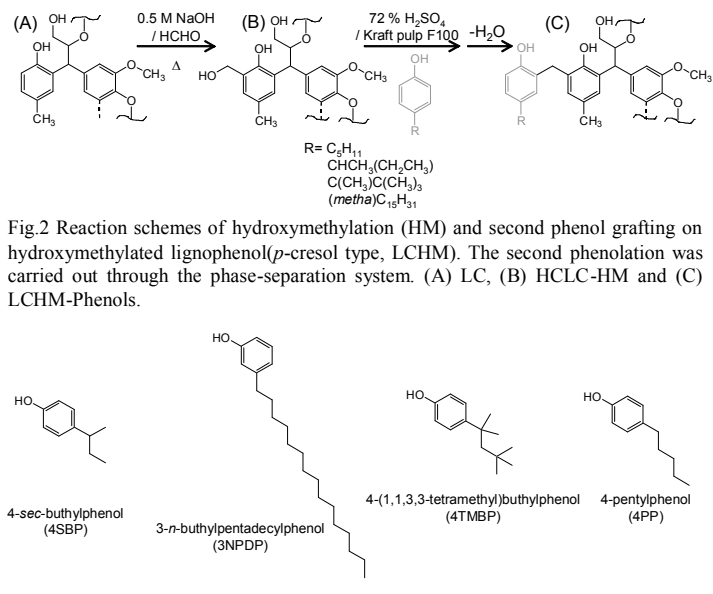

Fig. 3 Structures of (A) 4SBP, (B) 3NPDP, (C) 4TMBP and (D) 4PP

more well-designed products can be synthesized. Moreover various phenols, which have been considered that it is impossible to be grafted because of poor affinity with lignocellulosics, can be grafted to LP.

In this study, two grafting reactions were carried out. First, synthesis of blush-type LP derivatives by successive grafting of $p$-cresol via methylene chains was tried (Fig.2). Secondly, several alkyl phenols were tried to be grafted. Although direct grafting to lignin of several alkyl phenols such as $o-, m$-cresol, xylenols, ethylphenol, 4- $n$-propylphenol, guaiacol has been tried and succeeded already [3], more bulky phenols have not tried yet. Therefore four bulky alkyl phenols such as 4-sec-buthylphenol (4SBP), 3- $n$-pentadecylphenol (3NPDP), 4-(1,1,3,3-tetramethyl)buthyl phenol (4TMBP) and 4-pentylphenol(4PP) were tried to grafting on LPHM with vanishing reaction-supports under the phase-separation system (Fig.2, 3).

\section{EXPERIMENTAL}

2.1 Synthesis of lignophenols

Western Hemlock (Tsuga Heterophyll) and Hinoki cypress (Chamaecyparis obtusa) were used as softwood materials for the phase-separation system. The woody materials were milled for 80 mesh passed. Extractives in the material were removed by acetone at room temperature for $72 \mathrm{hrs}$. The milled woods $(500 \mathrm{~g})$ were thrown into acetone solution of $p$-cresol with concentration of $3 \mathrm{~mol} /$ phenylpropane units $\left(\mathrm{C}_{9}\right.$ units), which were subunits of native lignin determined by Klason method. After evaporation of acetone, $72 \%$ $\mathrm{H}_{2} \mathrm{SO}_{4}$ aq. solutions were poured into the material adsorbed with $p$-cresol at $30{ }^{\circ} \mathrm{C}$. Then the mixtures were stirred vigorously for $60 \mathrm{~min}$ soon after mixing. After 60 $\min$, the reaction mixtures were thrown into $20 \mathrm{~L}$ of de-ionized water with vigorously stirring by a homogenizer for $5 \mathrm{~min}$. Then the purple precipitations were washed until $\mathrm{pH}=5$. After drying, the precipitations were extracted by acetone. The acetone solutions were purified by dropped into EtOEt under vigorously stirring at $0{ }^{\circ} \mathrm{C}$. After evaporating and drying on $\mathrm{P}_{2} \mathrm{O}_{5}$, both Western Hemlock-lignophenol ( $p$-cresol type, LC1), and Hinoki cypress-lignophenol ( $p$-cresol type, HCLC1) were obtained [2-4].

2.2 Preparation of vanishing reaction-supports

Kraft pulp was dispersed in de-ionized water. The suspension was beaten to freeness 100 estimated using Canadian freeness-tester [12-13].

\subsection{Synthesis of derivatives}

Hydroxymethylated LC1(HMLC1) and HCLC (HM-HCLC1) were synthesized in $0.5 \mathrm{M} \mathrm{NaOH}$ solution by mixing $20 \mathrm{~mol}$ of formaldehyde for a amount of aromatic rings in lignophenol at $60{ }^{\circ} \mathrm{C}$ under $\mathrm{N}_{2}$ atomosphere with a stirring system and a reflux condenser. After $3 \mathrm{hrs}$ reaction, $1.0 \mathrm{M} \mathrm{HCl}$ was dropped into the mixture at $5{ }^{\circ} \mathrm{C}$ to $\mathrm{pH}=2.0$. The resulting precipitation was washed to $\mathrm{pH}=5.0$. After the insoluble residues were dried over $\mathrm{P}_{2} \mathrm{O}_{5}$, both HM-LC1 and HM-HCLC1 were obtained.

\subsection{Second phenol grafting}

Second phenol grafting lignophenol was synthesized from HM-LC1 or HM-HCLC1, with or without reaction-supports through the phase-separation system (Two-step method, process-I). $p$-Cresol, 4SBP, 4-TMBP, 3 NPDP and 4PP were used as the second grafting phenols. HM-LC1 $(0.15 \mathrm{~g})$ and phenols $\left(20 \mathrm{~mol} / \mathrm{C}_{9}\right)$ were adsorbed on $1.50 \mathrm{~g}$ of dry Kraft pulp (freeness 100) in $35.0 \mathrm{~mL}$ of THF. After evaporation of THF the resulting compound was reacted through the phase-separation system ( 2 step method, process-I) with $20.0 \mathrm{~mL}$ of $72 \% \mathrm{H}_{2} \mathrm{SO}_{4}$ under $30{ }^{\circ} \mathrm{C}$. After $30 \mathrm{~min}, 20.0$ $\mathrm{mL}$ of $p$-cresol was added to the reaction mixture as an extracting solvent under stirring. After $20 \mathrm{~min}$, the organic layer and aqueous layer were separated by centrifugation (3 $500 \mathrm{rpm}$ ). The organic layer was washed by EtOEt. Insoluble moiety was refined in the same way as LC1. After evaporation and dry on $\mathrm{P}_{2} \mathrm{O}_{5}$, lignophenol, the second phenol grafted (LC2) was obtained. Moreover only $p$-cresol type was repeated to be derived into LC3 and LC4.

\subsection{Characterization of lignophenols}

The structures of LP were characterized by Gel Permeation Chromatography (GPC), ${ }^{1} \mathrm{H}-\mathrm{NMR}$ and Thermal Mechanical Analysis (TMA). GPC was carried out by LC-10 system (Shimadzu Co.) with four columns (KF801, KF802, KF803 and KF804, Shodex Co.), using tetrahydrofuran (THF) after rectification as eluent. Both $M_{w}$ and $M_{n}$ were determined based on standard polystyrene. ${ }^{1} \mathrm{H}-\mathrm{NMR}$ spectrum was measured by FT-NMR500 (JASCO Co.) in $\mathrm{CDCl}_{3}$ or $\mathrm{CDCl}_{3} / \mathrm{C}_{5} \mathrm{D}_{5} \mathrm{~N}$ $=3 / 1(\mathrm{v} / \mathrm{v})$. TMA was also carried out by TMA-SS (SII Inc.) in the temperature range $50-280{ }^{\circ} \mathrm{C}$ at a rate of increase of $2{ }^{\circ} \mathrm{Cmin}^{-1}$ with vertical $49 \mathrm{mN}$ force, using penetrating technique for a measurement. UV-Vis spectroscopy was carried out on an UV-560 (JASCO Co.). FT-IR spectroscopy was also carried out on a FTIR8400 (Shimadzu Co.), using the $\mathrm{KBr}$ pellet technique for sample preparation. 
Table I Yields, weight average molecular weights and amounts of hydroxyl groups in lignophenols derived based on LC1(Western Hemlock-LC).

\begin{tabular}{|c|c|c|c|c|c|c|}
\hline \multirow[b]{2}{*}{ Lignophenols } & \multirow[b]{2}{*}{$\begin{array}{l}\text { Yield } \\
/ \%\end{array}$} & \multirow[b]{2}{*}{$M_{w}{ }^{\text {d) }}$} & \multirow[b]{2}{*}{$M_{w} M_{n}^{\mathrm{d})}$} & \multirow[b]{2}{*}{$\begin{array}{c}\text { Grafting } p \text {-cresole) } \\
\mathrm{mol} / \mathrm{C}_{9}\end{array}$} & \multicolumn{2}{|c|}{$-\mathrm{OH}^{\mathrm{e})}$} \\
\hline & & & & & $\begin{array}{l}\text { Phenol } \\
\mathrm{mol} / \mathrm{C}_{9}\end{array}$ & $\begin{array}{r}\text { Aliphatic } \\
\mathrm{mol} / \mathrm{C}_{9}\end{array}$ \\
\hline $\mathrm{LC1}^{\mathrm{a})}$ & $18.9^{\mathrm{c})}$ & 14200 & 2.8 & 0.76 & 1.11 & 0.95 \\
\hline LCHM1 b) & 98.7 & 15100 & 2.8 & 0.84 & 1.69 & 1.63 \\
\hline LC2 & 75.1 & 10600 & 1.8 & 1.10 & 1.86 & 1.16 \\
\hline LCHM2 & 98.2 & 10300 & 2.5 & 1.02 & 2.09 & 2.60 \\
\hline LC3 & 75.7 & 10800 & 1.9 & 1.65 & 2.13 & 1.61 \\
\hline LCHM3 & 92.3 & 11400 & 2.3 & 1.49 & 2.24 & 2.61 \\
\hline LC4 & 81.9 & 14400 & 1.9 & 1.92 & 2.20 & 0.92 \\
\hline
\end{tabular}

a) Western Hemlock-lignophenol ( $p$-cresol type), b) Hydroxymethylated LC1, c) based on native lignin in

\section{RESULTS AND DISCUSSION}

\subsection{Brush type derivatives}

LC1 was obtained with $60.9 \%$ and $18.9 \%$ yields based on wood meal and Klason lignin, respectively. As LC1 had $0.76 \mathrm{~mol} / \mathrm{C}_{9}$ of $p$-cresol, $0.24 \mathrm{~mol} / \mathrm{C}_{9}$ of un-reacted benzyl structures remained after reaction of the phase-separation system (Table I). After hydroxymethylation, LCHM1 was derived from LC1 with $98.7 \%$ yield and with $0.69 \mathrm{~mol} / \mathrm{C}_{9}$ of hydroxymethyl-OH in $1.63 \mathrm{~mol} / \mathrm{C}_{9}$ aliphatic-OH. Next, grafting reaction between LCHM1 and $p$-cresol on the surfaces of $p$-cresol / $72 \% \mathrm{H}_{2} \mathrm{SO}_{4}$ heterogeneous conditions resulted in $\mathrm{LC} 2$ with rich phenolic-OH. As $p$-cresol had affinity with lignophenol derivatives, reactions were carried out perfectly without reaction supports [12]. In these ways, both LC3 and LC4 were also synthesized. Interestingly, average molecular weights of both LC2 and LC3 were smaller than LC1 with low $M_{w} / M_{n}$ values. This implied that the un-reacted $\mathrm{C} \alpha$-ethers were cleft through the $2^{\text {nd }}$ and the $3^{\text {rd }}$ acid treatments. After cleavages of these linkages, generating small fractions were dissolved into EtOEt during purification processes. On the contrary, LC4 showed relatively high $M_{w}$ because there were few un-reacted arylethers already, therefore almost all $\mathrm{C} \alpha$ carbon were grafted by $p$-cresol. In fact, the yields of both LC2 and LC3 decreased, but only LC4 showed high yield. This indicated that after $4^{\text {th }} p$-cresol grafting via HM bridges there were few un-stable reaction sites in the molecules.

As shown in Table I, there were clear correlations between amounts of $p$-cresol and reaction frequencies. Moreover there were new signals of methylene proton at $4 \mathrm{ppm}$ in ${ }^{1} \mathrm{H}-\mathrm{NMR}$ (data not shown). These results indicated that successive linkages of $p$-cresol via methylene chains from $\mathrm{C} 1$-phenol nuclei. In addition to these results, TMA results showed LC2, LC3 and LC4

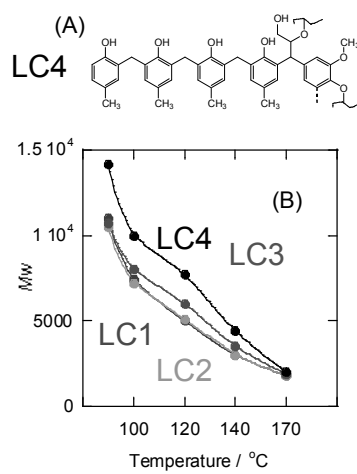

Fig.4 (A) Structures of brush-type derivatives (LC4) synthesized and (B) molecular weight variations after phenol switching treatments under $0.5 \mathrm{M} \mathrm{NaOH}$ aq. were plasticized near $150{ }^{\circ} \mathrm{C}$. Therefore these derivatives have relatively linear type polymeric structures.

Fig.4 demonstrated a structure of LC4 and drastic molecular weight variations after alkaline treatments. In general, lignophenols with ortho-OH phenols cleaved $\mathrm{C} \beta-\mathrm{O}-4$ main chains by nucreophillic attacks by lone-pairs on oxygen on phenoxide ions under alkaline condition over $100{ }^{\circ} \mathrm{C}[8,14-15]$. After the reactions, phenol structures were switched to counter parts. These reactions were based on neighbouring group participation [16] and arylmigration [17] of lignin-based materials. Interestingly, all LC materials showed convergence to $M_{w}=1000$. These results implied phenol-switching reactions occurred on the $1^{\text {st }}$ phenols grafted to $\mathrm{C} \alpha$ positions. Consequently, using HMLC as combination sites for phenols, new precise and practical applications with controls of both network density and branches are expected.

\subsection{Grafting of alkyl phenols using reaction-supports} In general, it has been difficult to use alkyl phenols with long or bulky chains as phenolic media for lignocellulosics through the phase-separation system, because of hydrophobicity. Due to this high hydrophobicity, alkyl phenols can not solvated native lignin. Without solvation of phenols, effective grafting reactions can not be occurred. Therefore only limited phenols with small alkyl substitutions (mainly under C3) were often used. If various phenols which have low affinity for lignocellulosics can be grafted into lignophenol molecules, possibility of lignophenols will be developed.

Both HCLC and HM-HCLC were obtained with high yields (Table II). In addition to these results, $p$-cresol contents were 0.74 and $0.79 \mathrm{~mol} / \mathrm{C}_{9}$, respectively. Although direct grafting reactions with HM-LC1 were tried, reactions have not occurred sufficiently with generating aggregations. On the other hand, using vanishing reaction-supports, solid of lignophenols with 4SBP, 4TMBPand 4PP were obtained but 3NPDP (Table II). Though some part of a derivative with 3NPDP was obtained as an acetone extraction, there was no solid of lignophenol derivatives after EtOEt or Benzene / $n$-Hexane purification systems. Both 4SBP and 4PP showed $47.6 \%$ and $59.4 \%$ yields, respectively.

Table II Results of synthesis of HCLC-HM-alkylphenols through the phase-separation system using Kraft pulp as vanishing reaction supports under r.t. and $1 \mathrm{~atm}$. Acid was $72 \% \mathrm{H}_{2} \mathrm{SO}_{4}$. Hydrophobic moieties were taken up by 10.0 $\mathrm{mL}$ of $p$-cresol after $30 \mathrm{~min}$ reaction. Extractions and purifications were carried out by both acetone and EtOEt.

\begin{tabular}{lcccc}
\hline Phenols ${ }^{\text {a) }}$ & Yield $^{\mathrm{c})} / \%$ & $M_{n}{ }^{\mathrm{i})}$ & $M_{w}{ }^{\mathrm{i})}$ & $M_{w} / M_{n}{ }^{\mathrm{i})}$ \\
\hline HCLC & 83.7 & 5670 & 21630 & 3.81 \\
HCLC-HM & 97.4 & 5040 & 19812 & 3.93 \\
\hline 4SBP & 47.6 & 5290 & 19270 & 3.64 \\
3NPDP ${ }^{\mathrm{d})}$ & $\mathrm{N} / \mathrm{A}$ & ----- & ---- & ---- \\
4TMBP & 25.4 & 4510 & 6030 & 1.34 \\
4PP & 59.4 & 10860 & 23080 & 2.12 \\
\hline
\end{tabular}

a) 4SBP: 4-sec-buthylphenol, 3NPDP: 3-n-pentadecylphenol, 4TMBP: $p$-(1,1,3,3tetramethylbuthyl) phenol, 4PP: 4-pentylphenol. c) HCLC-HM-3NPDP was dissolved in EtOEt. c) Yields were based on HCLC-HM, d) products did not obtained as a solid. 


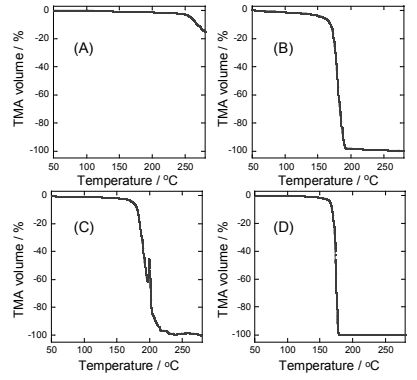

Fig.5 Thermal mechanical analyses (TMA) profiles for (A) HCLC-HM1, (B) HCLC-HM-4SBP, (C) HCLC-HMAs after EtOEt purification the aliquots showed light brown appearances, large parts of products would be dissolved like 3NPDP. As these phenols derived almost same $M_{w}$ as HCLC or HCLC-HM1, reactions between HM-groups and phenols seemed to be occurred sufficiently. But 4PP showed large $M_{n}$ probably due to polymerization at un-reacted $\mathrm{C} \alpha$-positions because these products were dissolved easily in acetone. Low yield of 4TMBP was due to both rich aggregations during acid treatments and high solubility in EtOEt. Therefore only small fraction of EtOEt insoluble fractions was obtained.

In addition to low polydispersities of 4SBP, 4TMBP and 4PP derivatives, results of TMA supported linear structures of resulting products. As shown in Fig.5, 4SBP, 4TMBP and 4PP derivatives showed clear solid-liquid transition points around $170{ }^{\circ} \mathrm{C}$, but HCLC-HM1 showed no plasticity. As there were few weight losses in HCLC-HM1 over $170{ }^{\circ} \mathrm{C}$ to $250{ }^{\circ} \mathrm{C}$ confirmed by thermal gravity analysis (TGA, data not shown), polymerization by heat-set was occurred. By intervals between start and end points of plasticity, information of viscosity was obtained. 4PP showed low viscosity but both 4SBP and 4TMBP showed relatively high viscosities. Moreover as Fig.5(C) showed there is a kick back type keen variation due to bubble up by gases such as $\mathrm{H}_{2} \mathrm{O}$ from liquid 4TMBP derivatives. These differences on viscosity were due to alkyl chains combined to $\mathrm{C} 1$ phenol via methylene chains. As HCLC showed clear plasticity around $170-180{ }^{\circ} \mathrm{C}$, main thermal properties were dominated by HCLC.

As shown in Fig.6, there were characteristic ${ }^{1} \mathrm{H}-\mathrm{NMR}$ spectra of 4SBP, 4TMBP and 4PP derivatives. In all spectra there were 8-6 ppm (aromatic ring-H), $5 \mathrm{ppm}$ (phenol-OH and un-reacted benzyl-H), 4 ppm
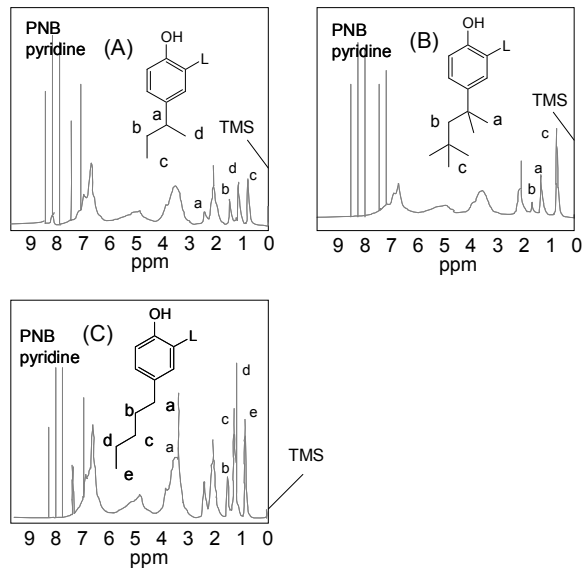

Fig.6 ${ }^{1} \mathrm{H}-\mathrm{NMR}$ spectra for (A) HCLC-HM-4SBP, (B) HCLC-HM-4TBMP and (C) HCLC-HM-4PP. L means lignin structures. (methylene bridges), 4-3 ppm (methoxyl groups), 2.5-2.0 ppm (methyl groups of $p$-cresol) and 2.5-0.5 ppm (characteristic alkyl groups) were observed. Unfortunately, it was impossible to caliculate amount of phenols or hydroxyl groups because signals corresponded to alkylchains were overwrapped over methyl signals of $p$-cresol. But these spectra showed there remained un-reacted benzyl groups with small amounts. Therefore it is necessary to improve efficiency on reactions by increasing stirring efficiency such as ultra sonic irradiations without heating [18].

\section{CONCLUSION}

Using hydroxymethylated lignophenols as reaction sites for second or more phenol grafting reactions were carried out. First, successive $p$-cresol grafting reactions produced brush type-derivatives, LC2, LC3 and LC4, with typical characteristic properties of LP such as thermal plasticity, solubility for solvents, phenolic properties and switching functionality. Due to high reactivity, syntheses using $p$-cresol have not required reaction supports. Secondly, grafting of alkylphenols such as 4SBP, 4TMBP and 4PP to HCLC-HM1 using pulps with freeness 100 as vanishing reaction supports were carried out. Resulting LPs showed properties of linear type polymers with $M_{w}$, thermal plasticities. Using these synthesis methods with benzyl structures mimic to the phase-separation system, developments on lignophenol molecules under precise control to generate novel functional materials are expected.

\section{REFERENCES}

[1] K. V. Sarkanen, C. H. Ludwig, "Lignins: Occurrence, Formation, Structure and Reactions", Wiley-Interscience, New York, 95, 165(1971)

[2] M. Funaoka, I. Abe, Tappi, 72, 145-155 (1989)

[3] M. Funaoka, M. Matsubara, N. Seki, S. Fukatsu, Biotechnology and Bioengineering, 46, 545-552(1995)

[4] M. Funaoka, Polym. Int, 47, 277-290 (1998)

[5] E. Adler, Wood Sci.Technol., 11, 169-218 (1977)

[6] M. Funaoka, M. Shibata, I. Abe, Holzforshung, 44, 357-366(1990)

[7] Y. Matsushita, S. Yasuda, Mokuzai Gakkaishi, 48, 55-62(2002)

[8] M. Funaoka, H. Ioka, T. Hosho, Y. Nagamatsu, Journal of Network Polymer Japan, 17, 121-129(1996)

[9] J. A. Hemmingson, G. J. Leary, W. A. Thomas, A. D. Woolshouse, J. Chem. Soc. Chem, Comm., 92-93(1978)

[10] J. A. Hemmingson, Aust. J. Chem., 32, 225-229(1979)

[11] G. J. Leary, D. A. Sawtell, H. Wong, Holzforshung, 37, 11-16(1983)

[12] M. Aoyagi, S. Yonekura, M. Funaoka, Trans. Mater. Res. Soc. J., 32, 1111-1114(2007)

[13]TAPPI-T227

[14]Y. Nagamatsu, M. Funaoka, SEN'I GAKKAISHI, 57, 54-59(2001)

[15] Y. Nagamatsu, M. Funaoka, SEN'I GAKKAISHI, 57, 82-87(2001)

[16] J. Gierer, C. R. Nelson, J. Org. Chem., 43, 4028(1978)

[17] J. Gierer, Wood Sci. Technol., 19, 289(1985)

[18] K. Nagamatsu, Y, Nagamatsu, M. Funaoka, Journal of Network Polymer Japan, 24, 30-39 (2003)

(Received December 12, 2008;Accepted September 16, 2009) 